Chapman University

Chapman University Digital Commons

Physical Therapy Faculty Articles and Research

Physical Therapy

$2-9-2021$

Effects of Auditory Cueing on Cadence and Gait Pattern

Andrew Carballo

Matthew Chang

Brian Hirmiz

Nicolette Lambright

Vivian Lee

See next page for additional authors

Follow this and additional works at: https://digitalcommons.chapman.edu/pt_articles

Part of the Other Rehabilitation and Therapy Commons, and the Physical Therapy Commons 


\section{Effects of Auditory Cueing on Cadence and Gait Pattern}

\section{Comments}

This is a pre-copy-editing, author-produced PDF of an article accepted for publication in the Proceedings of the Human Factors and Ergonomics Society Annual Meeting in 2020. The definitive publisherauthenticated version is available online at https://doi.org/10.1177/1071181320641293.

\section{Creative Commons License}

\section{(c) (1) (9)}

This work is licensed under a Creative Commons Attribution-Noncommercial-No Derivative Works 4.0 License.

\section{Copyright}

The authors

\section{Authors}

Andrew Carballo, Matthew Chang, Brian Hirmiz, Nicolette Lambright, Vivian Lee, Marla Lewis, Martin Nguyen, Matthew Gothong, and Rahul Soangra 


\title{
Effects of Auditory Cueing on Cadence and Gait Pattern
}

\author{
Andrei Carballo ${ }^{1}$, Matthew Chang ${ }^{1}$, Brian Hirmiz ${ }^{1}$, Nicolette Lambright ${ }^{1}$, Vivian Lee ${ }^{1}$, Marla Lewis ${ }^{1}$, \\ Martin Nguyen ${ }^{1}$, Matthew Gothong ${ }^{1}$, Rahul Soangra ${ }^{1 \& 2}$ \\ ${ }^{1}$ Crean College of Health and Behavioral Sciences, Chapman University, Orange CA 92866 \\ ${ }^{2}$ Fowler School of Engineering, Chapman University, Orange CA 92866
}

\begin{abstract}
A large portion of the population participate in gait rehabilitation, especially those with conditions such as increased fall risk such as stroke, or Parkinson's Disease. Some studies have shown that auditory cues help improve gait and reduce fall risk, but relationship with gait patterns is missing. In this study, eight participants walked at their preferred cadence and at increased and reduced cadence by $20 \%$. We found that step length and step width were not significantly different in all walking conditions. Decreased cadence resulted in an increase of swing time, stance time, stride time, and stance to swing ratio, and a decrease in stride length. Increased cadence resulted in a decrease in stance time, stride time, swing time, and stance to swing ratio, and an increase in stride length. The results suggest there is a strong correlation between auditory cues and gait patterns that can improve rehabilitative processes in the future.
\end{abstract}

\section{INTRODUCTION}

There is a rising level of interest in the increased number of fall risk patients. Previous research has shown that auditory stimulation, such as the use of a metronome or cueing, has a positive effect on gait. Characteristics of gait disorders in patients with Parkinson's Disease include shortened stride length and decreased walking speed. One specific study compared baseline cadence and cadence that was set to $10 \%$ above baseline. Cadence, or steps per minute, is a measurement used to assess a change in gait pattern. The study showed a decrease in gait cycle time and an increase in step length and step-extremity ratio, and the results confirm findings that auditory stimulation can be used to positively influence the gait of individuals with Parkinson's Disease (Freedland et al., 2012).

Stroke patients typically show reduced walking speed, decreased stride length, and cadence. Walking with auditory stimulation helps improve walking speed and stride length, thus optimizing cadence. People with sub-acute stroke, whose gait speed is increased by 0.16 meters per second are more likely to experience a reduction in disability. Studies have shown that walking training with cueing of cadence is more effective than walking training alone. There is a clear improvement in walking speed and stride length in those individuals using cueing. (Nascimento et. al, 2014). Fall risk can be attributed to many factors, such as age, and loss of coordination following a stroke. As one ages, their muscles atrophy, which can increase fall risk by slowing down gait speed. In such populations, fall risk is relatively high due to low gait stability. It has been found that rhythmic auditory stimulation may positively affect asymptomatic gait (Schreiber et. al, 2016). Changes to gait speed and walk ratio can also be altered with rhythmic auditory cueing such as a metronome (Ducharme et.al, 2018). Previous studies have also suggested that variance in walking components such as stride length and walking speed may contribute to stability (Fan et. al, 2016). Other studies have looked at the effects of varying gait cadences driven by rhythmic auditory cueing (Yu et. al, 2015).

\section{METHODS}

Subjects: Eight subjects with an average age of $24 \pm 2.72$ (years), body weight of $67.25 \pm 9.87(\mathrm{~kg})$, and height of

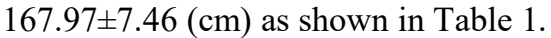

Process: Cadence was measured without influence of a metronome by documenting the time and number of steps taken to walk ten meters in an unobstructed walkway. This data provided normal cadence in steps per minute. The cadence $20 \%$ above and below subjects' normal cadence value was then calculated. Data was collected for two minutes at normal cadence, increased cadence, and decreased cadence. The order of conditions assigned was randomly predetermined. Several dependent variables were measured and taken into consideration for this experiment including gait phase lengths, number of steps to cross a predetermined distance, stride length variability and joint moments. Subjects walked on a self-paced treadmill with inertia sensors connected to Vicon Nexus software.

Equipment: Vicon Nexus, recorded data collected from 26 reflective sensors which were placed on each subject. Sensor locations were positioned on major anatomical landmarks. The Metronome Plus Application was set to one of three randomized cadences which included their normal and individualized increased and decreased cadence values. Rhythmic auditory cueing was administered to each participant through the metronome application in the predetermined randomized sequence. All eight participants walked for two minutes at each of their three cadence rates on the self-paced treadmill without using the handrails for balance assistance.

Data Processing: Personal data measurements such as the individual's age, height, weight, ankle and knee widths were entered into the Gait Offline Analysis Tool Software. The ankle and knee widths were measured using a caliper provided by the campus lab. Right gait cycles 39,40 , and 41 for each trial were of greatest interest in this experiment because the subject had become accustomed to the treadmill and set cadence to have a smoother gait pattern. An average of these three strides was taken to measure the parameters of gait at normal, increased, and decreased cadence values.

Additionally, Vicon Nexus produced experimental values for joint moments at the hip, knee and ankle for all three trials 
analyzed. JMP statistical analysis was used to analyze the data.

\section{RESULTS}

Overall, the subjects had significant differences $(p<0.03$ or $\mathrm{p}<0.0001$ ) in the left leg stance to swing ratio, stride time, stance time, stride length, and swing time in various conditions including low cadence, normal cadence, and high cadence. The step length and the step width measurements were not shown to be statistically significant in the data $(\mathrm{p}>$ $0.05)$. Data for subject two in the decreased cadence condition was omitted due to difficulties in data processing. As a result, the temporal parameters in the experiment proved to have a greater statistical significance in gait in comparison to most of the spatial parameters that did not change in response to the change of cadence.

Table 1. Anthropometric Measures of Subjects (mean \pm standard deviation).

\begin{tabular}{|c|c|c|}
\hline Age (years) & Height (cm) & Weight (km) \\
\hline $24 \pm 2.72$ & $167.97 \pm 7.46$ & $67.25 \pm 9.87$ \\
\hline
\end{tabular}

Table 2. Basic gait parameters of subjects (mean \pm standard deviation).

\section{CONDITION}

\begin{tabular}{|c|c|c|c|c|}
\hline & & $\begin{array}{l}\text { Increased } \\
\text { Cadence }\end{array}$ & $\begin{array}{r}\text { Decreased } \\
\text { Cadence }\end{array}$ & $\begin{array}{l}\text { Normal } \\
\text { Cadence }\end{array}$ \\
\hline $\begin{array}{c}\text { STANCE/SWING } \\
\text { (\% of Gait Cycle) }\end{array}$ & Mean & $63.31 \pm 1.374 *$ & $65.40 \pm 1.837$ & $64.48 \pm 1.377$ \\
\hline $\begin{array}{r}\text { STANCE TIME } \\
\text { (Seconds) }\end{array}$ & Mean & $0.574 \pm 0.043 *$ & $0.862 \pm 0.087 *$ & $0.690 \pm 0.060^{*}$ \\
\hline $\begin{array}{r}\text { STEP LENGTH } \\
\text { (Meters) }\end{array}$ & Mean & $0.724 \pm 0.093$ & $0.657 \pm 0.129$ & $0.692 \pm 0.094$ \\
\hline $\begin{array}{r}\text { STEP WIDTH } \\
\text { (Meters) }\end{array}$ & Mean & $0.134 \pm 0.032$ & $0.141 \pm 0.037$ & $0.136 \pm 0.038$ \\
\hline $\begin{array}{r}\text { STRIDE } \\
\text { LENGTH } \\
\text { (Meters) }\end{array}$ & Mean & $1.456 \pm 0.182^{\wedge}$ & $1.299 \pm 0.237^{\wedge}$ & $1.383 \pm 0.186$ \\
\hline $\begin{array}{r}\text { STRIDE TIME } \\
\text { (Seconds) }\end{array}$ & Mean & $0.906 \pm 0.055^{*}$ & $1.317 \pm 0.124 *$ & $1.069 \pm 0.078^{*}$ \\
\hline $\begin{array}{r}\text { SWING TIME } \\
\text { (\% of Gait Cycle) } \\
\end{array}$ & Mean & $0.332 \pm 0.016^{*}$ & $0.455 \pm 0.046 *$ & $0.379 \pm 0.024 *$ \\
\hline $\begin{array}{l}* \mathrm{p}<0.0001 \\
\wedge \mathrm{p}<0.03\end{array}$ & & & & \\
\hline
\end{tabular}

Stance to Swing Ratio: The stance phase occupied $63.31 \pm 1.374$ percent of the gait cycle at a faster cadence, in comparison to normal cadence when the stance phase was $64.48 \pm 1.377$ percent of the gait cycle. JMP indicated a significant difference in stance-swing ratio with faster cadence ( $p$ values $<0.0001$ ). There was no significant difference among the conditions of normal and low cadence in regard to stance to swing ratio (Fig 1, Table 2).

Stance Time: The stance time decreased with faster cadence and increased with slower cadence. When increasing the cadence by $20 \%$ of self-paced walk, the stance time decreased by $0.116 \pm 0.017$ seconds when compared to the normal cadence (Fig 2, Table 2). Decreasing the cadence resulted in the increase of stance time by $0.172 \pm 0.027$ seconds compared to normal cadence. The JMP indicated all conditions of cadence were different from one another in regard to stance time ( $p$ values $<0.0001$ ). Stride Length: The stride length of the participants appeared to increase with faster cadence and decrease with slower cadence. The average stride length was $0.724 \pm 0.093$ meters at high cadence, $0.692 \pm 0.094$ meters at normal cadence, and $0.657 \pm 0.129$ meters at low cadence. The JMP statistical analysis tool showed a significant difference between high cadence and low cadence ( $p$ values $<0.03$ ). Although, the high and low cadence conditions had no significant difference when individually compared to normal cadence (Fig. 3, Table 2). Stride Time: The stride time decreased with faster cadence and increased with lower cadence in order to remain at the same pace of the metronome. All three conditions of normal, high, and low cadence were found to be statistically different from one another ( $p$ values $<$ 0.0001 ). At lower cadence, the stride time increased by $0.247 \pm 0.046$ seconds and at higher cadence it decreased by $0.163 \pm 0.023$ seconds when compared to normal cadence (Fig. 4, Table 2). Swing Time: Similar to stance time, the swing time also decreased with faster cadence and increased with slower cadence. The swing time decreased by $0.047 \pm 0.008$ seconds when the cadence was increased by $20 \%$ and it increased by $0.076 \pm 0.022$ seconds when the cadence was decreased by $20 \%$ in comparison to normal cadence (Fig.5, Table 2). The JMP statistical analysis revealed that the conditions were all different from each other ( $\mathrm{p}$ values $<$ 0.0001).

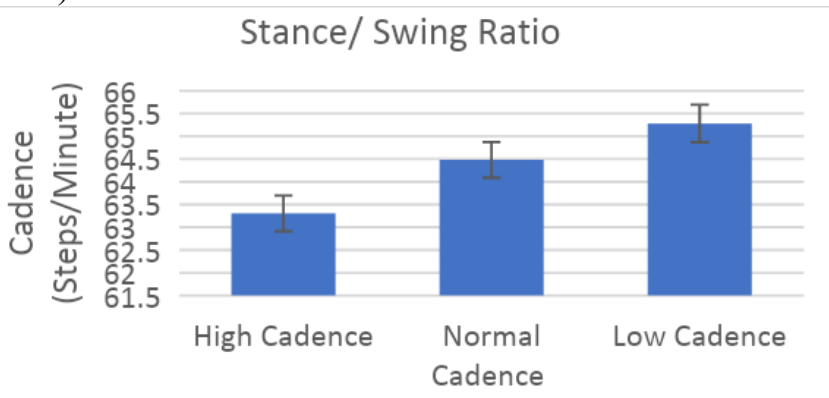

Figure 1. Stance/Swing Ratio Across Various Cadence Conditions

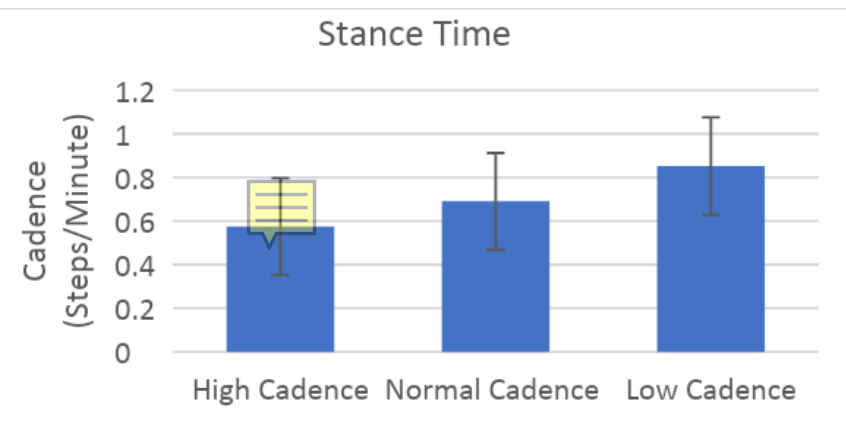

Figure 2. Stance Time Across Various Cadence Conditions 


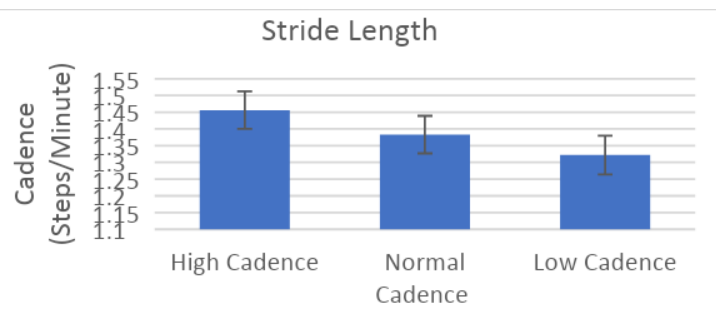

Figure 3. Stride length across various cadence conditions

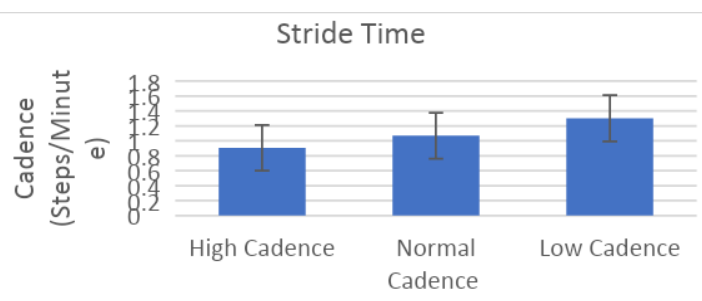

Figure 4. Stride Time Across Various Cadence Conditions

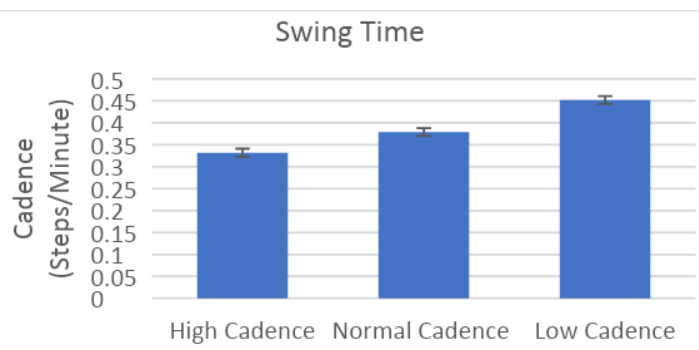

Figure 5. Swing Time Across Various Cadence Conditions Ankle Flexor Moment

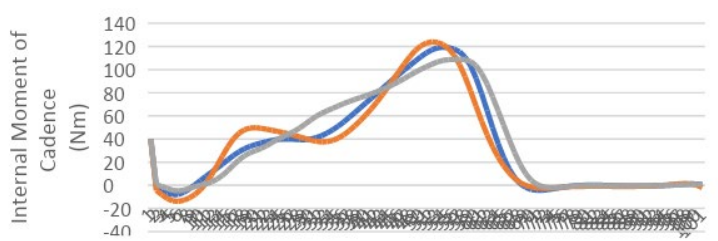

Percent of Gait Cycle (\%)

$-\quad-$

Figure 6. Ankle Flexor Moment Graph

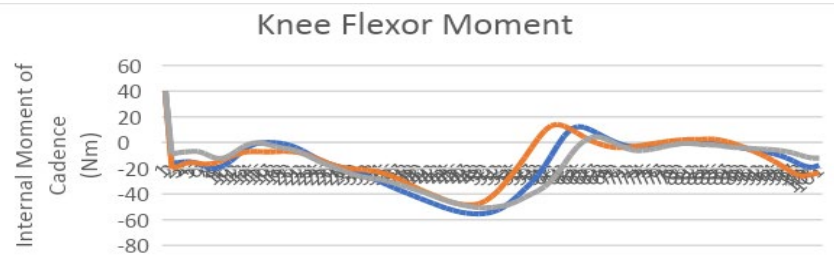

Percent of Gait Cycle (\%)

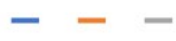

Figure 7. Knee Flexor Moment Graph

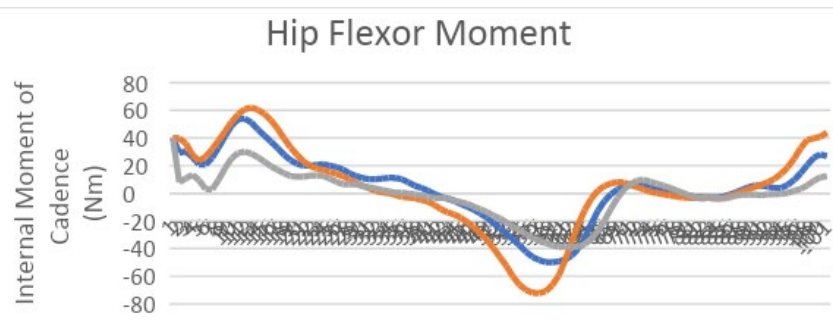

Peccent of Gait Cycle (\%)

Figure 8. Hip Flexor Moment Graph

DISCUSSION

The purpose of this study was to examine the effects of changing cadence on gait patterns. The results of this study suggest that the spatiotemporal parameters affected by the change in cadence were stance time, swing time, stride time, stride length, and stance to swing ratio. Whereas parameters, such as step length and step width, did not change with increase or decrease of cadence. The joint flexion moments of measured in this experiment and revealed that an increase in cadence results in larger flexion moments at all joints (Ardestani et al., 2016).

The gait parameters can be related to fall risk and may lead to insight on fall risk prevention. Participants were able to perform many full-length gait cycles under normal, high, and low cadence originally based on their self-paced speed. The stance to swing ratio decreased when a faster cadence was implemented. As a result, the swing phase began earlier in the gait cycle. This possibly occurred to allow more time for leg progression when walking at an increased cadence (Hebenstreit et al., 2015). In addition to the stance to swing ratio, the stance time and swing time variables also changed at different cadences. This important finding supports that a decrease in gait cycle time at an increased cadence can aid in gait rehabilitation of patients with Parkinson's disease. The introduction of the increased cadence can speed up the walk of a patient and alleviate symptoms, such as slow step time and shuffling of feet, which are symptoms related to Parkinson's disease (Kwon et al., 2017).

This study revealed that temporal parameters had statistically significant differences as they pertained to changes in cadence speeds. The spatial parameters did not appear to be statistically significant with the exception of the stride length at high and low cadences (Table 2). This finding is contradictory to the step length data as it would be expected that a stride would be measured at double the step distance for symmetrical gait. Two possible explanations have been suggested to explain this finding. The first is that the small sample size was not be capable of detecting statistically significant differences in step length, providing inconclusive data. The second is at higher and lower cadences, the stride length is different due to an asymmetric gait pattern as only the data for one leg was analyzed in these results. Participants attmepted to match their steps to the rhythmic auditory cueing therefore it is possible that the dominant leg was utilized to match their steps to the beat of the metronome. For this reason, the step length of the dominant leg would be 
consistent, however the full stride time takes into account the motion of the opposite leg which may be responsible for producing the statistically significant differences in stride length.

The joint kinetics were measured at normal, high, and low cadences for the hip, knee, and ankle joints during a complete gait cycle for one subject. The increased cadence condition created the largest moments in all joints (Ardestani et al., 2016). In the ankle joint moment, as the cadence increased, more plantar flexor moments were created (Fig. 6). The additional plantar flexor moment occurred in the mid stance period of the gait cycle and the other plantar flexor moment occurred at the end of terminal stance and beginning of preswing period, where one would expect to see a plantar flexor moment of the ankle. At normal and slower cadence, the graph has one single wave, which suggests there is one plantar flexor moment during the complete gait cycle. As it pertains to the knee, the graph shows little variation in knee flexor moment when the cadence is altered (Fig. 7). For this particular individual, there is also a lack of an extensor moment during the loading response and midstance period of the gait cycle when compared to a standard graph of joint flexion moments at the knee. This is found in all conditions of the study including normal cadence, high cadence, and low cadence. Finally, the hip joint flexor moment was affected by the cadence condition. With increased cadence, larger flexor and extensor moments of the hip are presented by the peaks on the graph (Fig. 8). Whereas decreased cadence saw the flexor and extensor moments of the hip become smaller in comparison to the moments at normal cadence (Fig 8).

The increase in joint moments with increased cadence alter the biomechanics of the lower limb and can lead to faster progression of osteoarthritis (Ardestani et al., 2016). This may become clinically relevant in gait rehabilitation strategy in treating patients. Small changes in the pace at which patients walk may reduce the risk of joint pathology (Ardestani et al., 2016). In addition, it is important to consider the changes in joint moments in decreased cadence. Decreased cadence, the peak joint moments are reduced, as well as the joint power generation due to the decrease in walking speed (Browne \& Franz, 2017). Less joint power generation may slow down the process of potential osteoarthritis. Therefore, older patients may be advised and trained to walk at a slower speed during gait training to prevent onset of osteoarthritis.

Interestingly, the Borg Scale of Perceived Exertion has not been widely utilized in conjunction with this research. If heart rate and blood pressure data was incorporated, the study could show how cadence affects patients on a physiological level in addition to the kinematic components. The importance of fatigue is particularly relevant for physical therapy as it affects patients' willingness to participate in assigned exercise activities. Recognizing that the elderly may experience fatigue earlier in exercise, it is important to consider that this may affect their attitudes toward therapy (Egerton, et. al, 2009). This study may provide additional clinical implications for physical therapy upon extrapolating the relationship between the applied cadence and aerobic fitness of the patient through indirect VO2 calorimetry measurements (Peacock, et. al, 2014). Based on this study, a faster cadence dictates the patient's stride time and gait pattern, thus an auditory cueing exercise could be included when prescribing a physical therapy plan. Recommending a patient to exercise at a given pace may help the therapist to better control the patient's activities and energy expenditure for their specific condition. This may be important for clients recovering from surgery to ensure that adequate exercise is administered without inadvertently causing too much stress or exertion.

A majority of the participants recalled having the most difficulty with adhering to the slower metronome upon reflection of the experiment. The human body seeks to take mechanical advantage of joint moments and momentum to continue to propel itself in a forward direction. For this reason, excessive energy and concentration needs to be exerted to slow one down from their natural gait. Walking at a slower pace could potentially increase fall risk in elderly patients or those with preexisting conditions related to balance deficiencies (Freedland et al., 2012). In addition, a rapid decline trajectory for gait speed corresponded to higher mortality risk compared to a constant healthy trajectory (White et al., 2012). The correlation between base gait speed and mortality provides strong evidence that walking pace is critical to maintaining good health even in old age. As this study has shown, an individual's walking speed does increase given faster auditory cueing. For therapists, it may be beneficial to incorporate such rhythmic cadence training in exercise programs for their patients to ensure they enjoy healthy and longer lives.

\section{REFERENCES}

Ardestani M.M., Ferrigno C., Moazen M., Wimmer M.A. (2016). From normal to fast walking: Impact of cadence and stride length on lower extremity joint moments. Gait \& Posture, 46,118-125. doi:10.1016/j.gaitpost.2016.02.005

Browne M.G., Franz J.R. (2017). The independent effects of speed and propulsive force on joint power generation in walking. Journal of Biomechanics, 55,48-55.

doi:10.1016/j.jbiomech.2017.02.011

Ducharme S.W., Sands C.J., Moore C.C., Aguiar E.J., Hamill J., Tudor-Locke C. (2018). Changes to gait speed and the walk ratio with rhythmic auditory cueing. Gait \& Posture. 66, 255259. doi:10.1016/j.gaitpost.2018.09.006.

Egerton T., Brauer S.G., Cresswell A.G. (2009). Fatigue after physical activity in healthy and balance-impaired elderly. Journal of Aging and Physical Activity, 17(1), 89-105.

Fan Y., Li Z., Han S., Lv C., Zhang B. (2016). The influence of gait speed on the stability of walking among the elderly. Gait Posture, 47, 31-36. doi:10.1016/j.gaitpost.2016.02.018

Freedland R.L., Festa C., Sealy M., et al. (2002). The effects of pulsed auditory stimulation on various gait measurements in persons with Parkinson's Disease. NeuroRehabilitation, $17(1), 81$. 
Hebenstreit F., Leibold A., Krinner S., Welsch G., Lochmann M., Eskofier B.M. (2015). Effect of walking speed on gait sub phase durations. Human Movement Science, 43, 118124. doi:10.1016/j.humov.2015.07.009

Kwon K.Y., Lee H.M., Kang S.H., Pyo S.J., Kim H.J, Koh S.B. (2017). Recuperation of slow walking in de novo Parkinson's disease is more closely associated with increased cadence, rather than with expanded stride length. Gait \& Posture, 58, 1-6. doi:10.1016/j.gaitpost.2017.06.266

Nascimento L.R., de Oliveira C.Q., Ada L, Michaelsen S.M., Teixeira-Salmela L.F. (2015). J Physiother, 61(1), 10-5. doi:10.1016/j.jphys.2014.11.015. Epub 2014 Dec 17.

Review.

Peacock L., Hewitt A., Rowe D.A., Sutherland R. (2014). Stride Rate and Walking Intensity in Healthy Older Adults. Journal of Aging \& Physical Activity, 22(2), 276-283. doi:10.1123/JAPA.2012-0333.

Roerdink Melvyn., J.M. Bank Paulina, C.E. Peper, Beek Peter $\mathrm{J}$ (2010). Practical implications for the use of acoustic rhythms in gait rehabilitation. Gait \& Posture, 33, 690-694.

doi.org/10.1016/j.gaitpost.2011.03.00.

Schreiber C., Remacle A., Chantraine F., Kolanowski E., Moissenet F. (2016). Full length article: Influence of a rhythmic auditory stimulation on asymptomatic gait. Gait \& Posture, 50, 17-22. doi:10.1016/j.gaitpost.2016.07.319.

Suh J.H., Han S.J., Jeon S.Y., Kim H.J., Lee J.E., Yoon T.S., and Chong H.J. (2014). Effect of Rhythmic auditory stimulation on gait and balance in hemiplegic stroke patients. Neurorehabilitation, 34(1), 193-199. Doi: 10:3233/NRE131008.

White D.K., Neogi T., Nevitt M.C., Peloquin C.E., Zhu Y., Boudreau R.M., Cauley J.A., Ferrucci L., Harris T.B., Satterfield S.M., Simonsick E.M., Strotmeyer E.S., Zhang Y. (2012). Trajectories of gait speed predict mortality in wellfunctioning older adults: the Health, Aging and Body Composition study. J Gerontol A Biol Sci Med Sci, 68(4):45664.

Yu L., Zhang Q., Hu C., Huang Q., Ye M., Li D. (2015). Effects of different frequencies of rhythmic auditory cueing on the stride length, cadence, and gait speed in healthy young females. Journal of Physical Therapy Science, 27(2), 485-487. doi:10.1589/jpts.27.485 